\title{
Classification of Multispectral Satellite Images using Clustering with SVM Classifier
}

\author{
S.V.S Prasad \\ Department of ECE, \\ MLR Institute of Technology, \\ Hyderabad, A.P, India.
}

\author{
Dr. T. Satya Savitri \\ Department of ECE, \\ JNTU College of Engg, \\ Hyderabad, A.P, India.
}

\author{
Dr. I.V. Murali Krishna \\ Department of SIT, \\ JNTU College of Engg, \\ Hyderabad, A.P, India
}

\begin{abstract}
Multi-spectral satellite imagery is an economical, precise and appropriate method of obtaining information on land use and land cover since they provide data at regular intervals and is economical when compared to the other traditional methods of ground survey and aerial photography. Classification of multispectral remotely sensed data is investigated with a special focus on uncertainty analysis in the produced landcover maps. Here, we have proposed an efficient technique for classifying the multispectral satellite images using SVM into land cover and land use sectors. In the proposed classification technique initially pre-processing is done where the input image is subjected to a set of pre-processing steps which includes Gaussian filtering and RGB to Labcolorspace image conversion. Subsequently, segmentation using fuzzy incorporated hierarchical clustering technique is carried out. Then training of the SVM is carried out in the training data selection procedure and finally the classification step, where the cluster centroids are subjected to the trained SVM to obtain the land use and land cover sectors. The experimentation is carried out using the multi-spectral satellite images and the analysis ensures that the performance of the proposed technique is improved compared with traditional clustering algorithm
\end{abstract}

Keywords: Multispectral satellite image, Clustering, Classification, Support vector machine.

\section{INTRODUCTION}

Multispectral image delivers a great source of data for studying spatial and temporal changeability of the environmental factors. It can be utilized in a number of applications which consists of reconnaissance, making of mapping products for military and civil use, assessment of environmental damage, nursing of land use, radiation level check, urban planning, growth directive, soil test and crop outcome increment [8]. One major area where we use multispectral image is in the process of classification and mapping of vegetation over large spatial scales, as the remote sensing data delivers very good coverage, mapping and classification of land cover features like vegetation, soil, water and forests. This behaves like a replacement for the normal classification techniques, which necessitates expensive and time-intensive field surveys [10]. Researches and studies on image classification have long been fascinated the concentration of the scientific community, from the time when many environmental and socioeconomic presentations are based on the classification consequences [9]. Usually, a classification system makes a classification map of the identifiable or meaningful features or classes of land cover sections in a part [11]. Regardless of all the advantages, classification of land-cover using multispectral imagery is a difficult subject because of the complexity of landscapes and the spatial and spectral resolution of the images being engaged.

Multispectral images consist of info collected over a wide range of changes on frequencies and these frequencies change over different areas (irregular or frequency variant behaviour of the signal) [15]. The overall complex nature of multispectral image data can be attributed to the spectral characteristics with correlated bands and spatial features related within the same band which is also known as the spatial correlation. An efficient method capable of arranging the spectral and spatial (contextual) info existing in the multispectral data can increase the accuracy level of the classification in a good way when matched with the traditional non-contextual information based techniques. Researches and studies on multispectral image classification have long acquired the attention of the scientific community, since most environmental and socio-economic applications are based on the classification results [9].

Multispectral image classification can be considered as a combined project of both image processing and classification methods. Usually, image classification, in the process of remote sensing is the method of referring pixels or the basic units of an image to the classes. It is mostly likely to create groups of similar pixels found in image data into classes that match the informational categories of user interest by matching the pixels to one another and to those of the said identity [12]. Many techniques of image classification have been introduced and numerous areas like image analysis and pattern recognition use the vital term, classification. In many circumstances, the classification itself may become the entity of the analysis and serve as the ultimate matter. In other scenarios, the classification aims to be the middle step in more complicated computations, such as land- degradation studies, process studies, landscape modeling, coastal zone management, resource management and other environment monitoring applications. Due to this, image classification has grown and established as a major tool for learning digital images. Furthermore, the choice of the ideal classification method to be used can have a considerable effect on the outcomes of it. The classification is used as a major product or as one of many computational methods used for deriving info from an image for further learning [12].

The available literature has a good number of supervised techniques that have been created to overcome the multispectral data classification problematic scene. The statistical technique used for the earlier studies of landcover classification is the maximum likelihood classifier. In recent times, various studies have applied artificial intelligence techniques as seconds to the remotely-sensed 
image classification applications [13]. Besides, different ensemble classification technique has been presented to increase the classification precision [14]. Scientists have made great strides in creating efficient classification systems and methods for increasing the classification accuracy.

The main focus of this research is to classify into land use and the land cover. Land cover denotes to characteristic features of land surface. These can be natural, semi-natural, managed or totally man-made and are directly observable. The major motive for making land cover maps is to give us a clear picture of the stock and state of our natural and built resources. A land cover classification is vital ingredient in creating a responsible attitude to environmental management. Land cover is different from land use even though the two terms are commonly being used interchangeably. Land use is a statement of how people utilize the land and socio-economic activity-urban and agricultural land uses are two of the frequent commonly used high-level classes of use. At some point or place, there can be more than one alternate land uses, the description of which may have a political dimension. Land cover classifications are major inputs to environmental and land use planning at local, regional, and national levels [5]. Classification of multispectral remotely sensed data is computed with a special attention on uncertainty computation in the land-cover maps. Here, we have proposed an efficient technique for classifying the multispectral satellite images into land cover and land use sectors using SVM. The proposed classification technique comprises of four phases which includes pre-processing, segmentation using clustering technique, training data selection for SVM and classification using trained SVM. Multispectral images cannot be fed directly into the SVM for training and testing. The input image is subjected to a set of pre-processing so that the image gets transformed suitably for segmentation. Then, we use fuzzy incorporated hierarchical clustering algorithm for segmentation of the image into clusters. Then, the cluster centroids are then subjected to trained SVM and the final classification of the multispectral satellite images into land use and land cover is obtained.

The rest of the paper is organized as follows: A brief review of researches related to the proposed technique is presented in section 2. The background of Support Vector Machines (SVM) is detailed in section 3. A brief description of the utilized algorithms and concepts are given in section 4 . The proposed classification technique is presented in Section 5. The experimental results and discussion are given in Section 6. The conclusions are summed up in Section 7.

\section{REVIEW OF RELATED WORKS}

A lot of research works have been carried out in the literature for remotely sensed multispectral image classification and some of them have motivated us to take up this research. Brief reviews of some of those recent significant researches are presented below:

$\mathrm{K}$ Perumal and R Bhaskaran [1] proposed a proficient land use image classification system with the help of image processing methods and Support Vector Machines. The proposed method consisted of SVM Training and then, SVM Testing. In the training part, the multispectral image data was done unsharp filtering and nonlinear isotropic diffusion segmentation. The segmented image pixels matching the land use regions were then given as training input to the SVM. And in order to have the testing in an automatic manner, the regions segmented by nonlinear isotropic diffusion segmentation were then mined out with the use of the active contour model. Then, the trained SVM precisely classified the land cover regions based on the pixel values of the mined out area. The experimental results showed the efficacy of the proposed classification technique in classifying land cover regions.

Jan Knorn et al. [2] presented a technique for the Landsat image classification. Their goal was to remove the drawbacks of a normal system and to examine the chain classifications, which is to the classify Landsat images based on the info in the overlapping regions of nearby sights. The SVMs classified 8 sight scenes with a precision in the range of $92.1 \%$ and $98.9 \%$. Xiaochen Zou and Daoliang $\mathrm{Li}$ [3] proposed an outline of a number of different techniques to image texture analysis. All the outcomes of the classifications were matched and computed. In their work, they used grey level co-occurrence matrix (GLCM) and the feature label images, which assisted in the classification of remote sensing.

Reda A. El-Khoribi [4] proposed a method to perform classification of multispectral images in which, a discriminative training procedure for discrete hidden Markov tree (HMT) productive structures were given to the multi-resolution ranklet changes. and it was performed and evaluated on a set of Landsat 7-band images and used the acceptable statistics of the HMT generative model. B Sowmya and B Sheelarani [5] clarified the mission of land cover classification making use of the renewed fuzzy C means. In order to assess the image on all of its colours, the likely colours were grouped together by the renewed fuzzy $\mathrm{C}$ means algorithm. The segmented images were matched using image quality evaluation metrics which used peak signal to noise ratio (PSNR), error image and compression ratio. The time needed for image segmentation was also used as an assessment factor.

V.K.Panchal et al. [6] presented a technique in which concentrated on the classification of the satellite image of a specific land cover making use of the concept of Biogeography based Optimization. Alterations were applied to the original $\mathrm{BBO}$ algorithm to incorporate clustering and the modified changed algorithm was employed to classify the satellite image of the given area. Highly precise land cover features were mined successfully when the proposed technique was made use of. Huang B et al. [7] presented a SVM modeling framework to discuss and assess the landuse change in relation to different factors such as population, distance to roads and facilities, and surrounding land use. An unbalanced SVM was implemented by improving the standard normal SVMs in order to solve the issues faced by normal SVM, such as having an unstable land-use data.

\section{SUPPORT VECTOR MACHINE}

Support Vector Machines (SVM) [16] is a statistical learning based classification system. The SVM sections the classes with respect to a decision surface that maximizes the margin between the classes. The surface is normally known as the optimal hyperplane and the data points closest to the optimal hyperplane are known as the support vectors. These support vectors are the most important elements of the training set. Some deviations of SVM are: 1) the SVM can be modified to make it a nonlinear classifier by the employment of nonlinear kernels and 2) a multiclass 
classifier can be made by clubbing a large number of binary SVM classifiers (making a binary classifier for every possible pair of classes). For multiclass classification, the pair wise classification strategy is regularly made use of. The result of the SVM classification is the decision values of each pixel for each of the class. This is employed for probability estimates [17].

In the two-class scenario, a support vector classifier produces a try to attain a hyperplane that minimizes the distance from the members of each class to the optional hyperplane. A two-class classification problem can be defined in the following way: Suppose there are M training samples that can be given by the set pairs

$$
\left.\left\{\left(x_{i}, y_{i}\right), i=1,2,3 \ldots \ldots, M\right)\right\} \text { with } \quad x_{i} \text { being the }
$$

class label of value \pm 1 and $y_{i} \in{ }^{n}$ where feature vector with $\mathrm{n}$ components. The classifier is given by the function $f(y ; \alpha) \rightarrow x$ with $\alpha$, the parameter factors of the classifier. The figure 1 shows the Maximum-margin hyperplane and margins for an SVM trained with samples from two classes. Samples on the margin are called the support vectors.

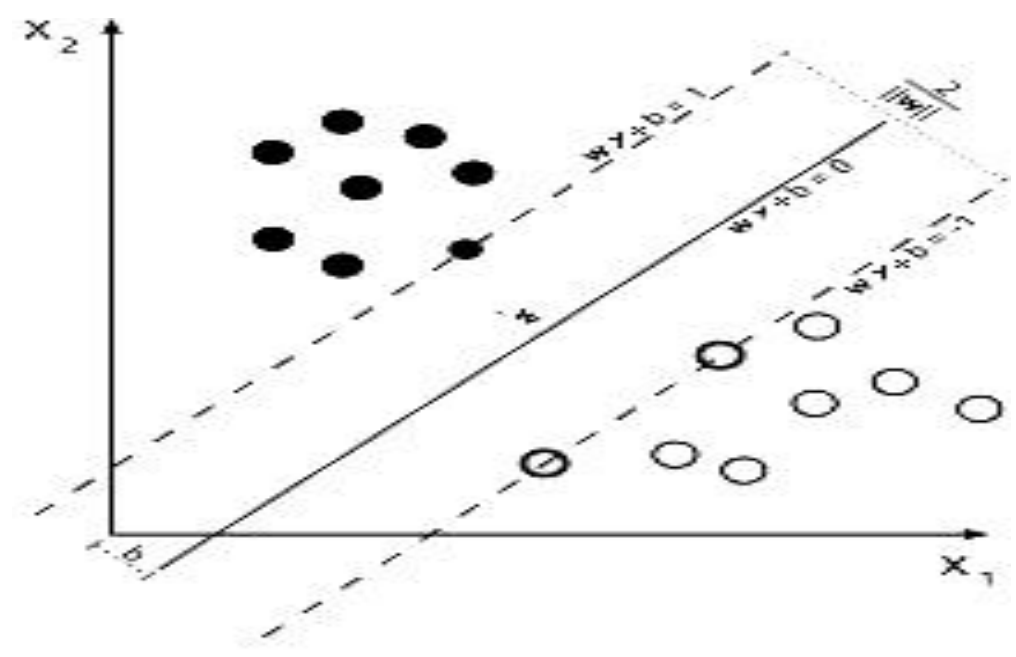

Figure 1. Maximum-margin hyperplane and margins for an SVM trained with samples from two classes.

An optimum separating hyperplane is found out by the SVM algorithm such that:1) Samples with labels \pm 1 are situated on each side of the hyperplane; 2) The distance of the nearest vectors to the hyperplane in each side of maximum are called support vectors and the distance is the optimal margin. The hyperplane is given by the equation by $w \cdot y+b=0$ where $(w, b)$ are the parameter factors of the hyperplane. The vectors that are not on this hyperplane lead to $w \cdot y+b>0$ and let the classifier to be given as $f(y ; \alpha)=\operatorname{sgm}(w \cdot y+b)$. The support vectors lie on two hyperplanes, which are parallel to the optimal hyperplane, of equation $w \cdot y+b= \pm 1$. The maximization of the margin with the equations of the two support vector hyperplanes contributes to the following constrained optimization $\quad$ problem $\quad \min \frac{1}{2}\|w\|^{2} \quad$ with $x_{i}(w \cdot y+b) \geq 1, i=1,2, \ldots \ldots ., M$.

\section{DESCRIPTION OF BASIC ALGORITHMS UTILIZED}

For the purpose of effectively classifying the satellite image into the land use and land cover regions, we make use of concepts of Hierarchical clustering, Fuzzy C Means algorithms in our proposed technique. These algorithms are discussed in this section.

\subsection{Hierarchical Clustering Algorithm}

Hierarchical algorithms are of two types, one is the agglomerative and the other, divisive. Hierarchical clustering [18] delivers a natural choice to graphically embody the dataset. However, it has disadvantages of being highly complex and also the fact that, a minor variation in the dataset may greatly vary the hierarchical dendrogram structure. Here we make use of agglomerative approach in our proposed method for cancer classification.

When a set of $\mathrm{N}$ items is given as the input that has to be clustered and it produces a $\mathrm{N}^{*} \mathrm{~N}$ distance matrix and the basic procedure of hierarchical clustering developed by S.C Johnson [18] have the steps given below,

1. Start the process by committing each item to a cluster, so that if there are $\mathrm{N}$ items, there will be $\mathrm{N}$ clusters, each cluster having one item each. Here the distances (similarities) between the clusters will be the same as the distances (similarities) between the items they include.

2. Find the nearest (most similar) pair of clusters and combine them into a single cluster, so that it will result in one cluster less.

3. Calculate distances (similarities) between the newly formed cluster and each of the old clusters.

4. Repeat steps 2 and 3 until all items are clustered into a final single cluster of size $\mathrm{N}$. 
The main drawback of agglomerative clustering technique is the fact that they do not scale well as the time complexity is at least $\mathrm{O}(\mathrm{n} 2)$, where $\mathrm{n}$ is the number of total items. That is the time incurred is high especially when a large set of data is considered.

\subsection{Fuzzy C Means Clustering}

Fuzzy c-means (FCM) [19] is a technique of clustering which permits one piece of data to two or more clusters. This technique was introduced by Dunn in 1973 and renewed by Bezdek in 1981 and it is mostly employed in pattern recognition.

It is based on minimization of the following objective function:

$$
\begin{gathered}
J_{m}=\sum_{i=1}^{N} \sum_{j-1}^{C} \mu_{i j}^{m}\left\|x_{j}-c_{j}\right\|^{2} \quad 1 \leq m<\infty \\
\text { where } m \text { is any real number greater than } \\
1, \mu_{i j} \text { is the degree of membership of } x_{i} \text { in the }
\end{gathered}
$$

cluster $j, x_{i}$ is the $i$ th of d-dimensional measured data, $c_{j}$ is the d-dimension center of the cluster, and $\|*\|$ is any norm expressing the similarity between any measured data and the center.

Fuzzy partitioning is carried out through an iterative optimization of the objective function shown above, with the update of membership $\mu_{i j}$ and the cluster centers $c_{j}$ by:

$$
\begin{gathered}
\mu_{i j}=\frac{1}{\sum_{k=1}^{C}\left(\frac{\left\|x_{i}-c_{i}\right\|}{\left\|x_{i}-c_{k}\right\|}\right)^{\frac{2}{m-1}}} \\
c_{j}=\frac{\sum_{i=1}^{N} \mu_{i j}^{m} x_{i}}{\sum_{i=1}^{N} \mu_{i j}^{m}}
\end{gathered}
$$

This iteration will stop when his iteration will stop when $\max _{i j}\left\{\left|\mu_{i j}^{k=1}-\mu_{i j}^{k}\right|<\xi\right\}$, where $\xi$ is a termination criterion between 0 and 1 , whereas $k$ is the iteration step. This procedure converges to a local minimum or a saddle point of $J_{m}$.

The main drawback with the use of traditional FCM for the clustering process is the fact that it does not yield the accurate results. That is every time FCM is performed on the same data, we may get varying results.
In our proposed method, we propose a Fuzzy incorporated Hierarchical clustering technique which improves on the drawbacks of both the hierarchical clustering and the FCM. That is time incurred by the proposed technique is less when compared to the normal hierarchical clustering and also the accuracy level goes up when compared to the normal FCM which makes the proposed technique a superior one.

\section{PROPOSED TECHNIQUE FOR CLASSIFICATION MULTISPECTRAL OF IMAGES USING CLUSTERING WITH SVM CLASSIFIER}

This section presents the proposed technique of classification of multispectral satellite images using clustering with SVM classifier. Initially in our proposed classification technique, pre-processing is done where the input image is subjected to a set of pre-processing steps such as Gaussian filtering and conversion of RGB to Lab colour space image so that the image gets transformed suitably for segmentation. The pre-processed image is segmented using the fuzzy incorporated hierarchical clustering algorithm. Training data selection is carried out for SVM and finally, classification of the multispectral satellite images using SVM is done based on the trained data and the centroid pixel values. The block diagram of the proposed technique is given in the figure 2 below.

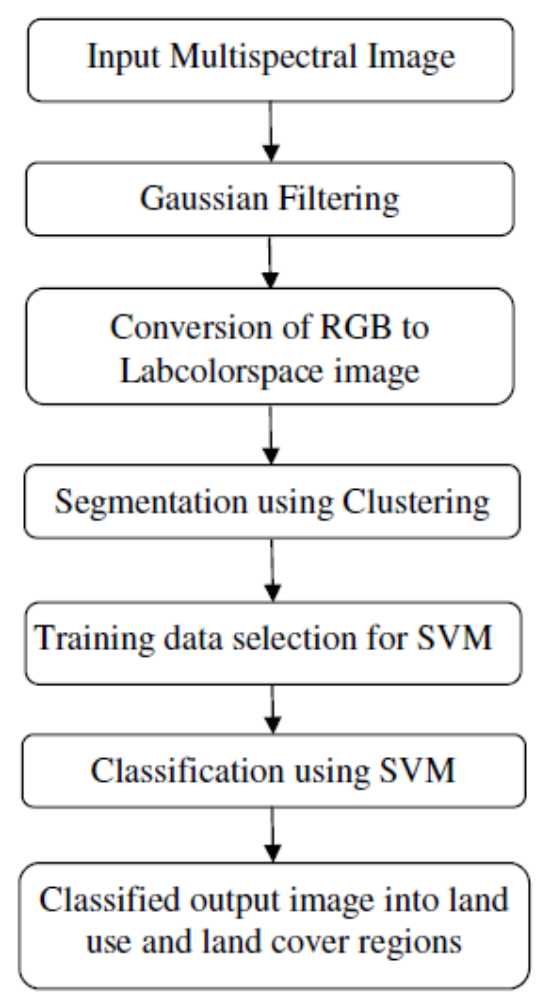

Figure 2. Block diagram of the proposed technique 


\subsection{Pre-processing}

Multispectral images cannot be fed directly into the SVM for training and testing. The input multispectral satellite image is subjected to a set of pre-processing steps so that the image gets transformed suitably for the further processing. Here we employ two step preprocessing procedure in which first the input image is passed through a Gaussian filter to reduce the noise and get a better image fit for segmentation. Passing the image through the Gaussian filter also enhances the image quality. In the second step in the preprocessing, we convert the image from the RGB model to Lab colour space Image which makes the more fit to be segmented by the use of clustering technique.

A. Gaussian Filter: A Gaussian filter [20] is a filter whose impulse response is a Gaussian function. Gaussian filters are developed avoid overshoot of step function input while reducing the rise and fall time. This character is very much linked to the fact that the Gaussian filter has the minimum possible group delay. In mathematical terms, a Gaussian filter changes the input signal by convolution with a Gaussian function; this change is also called the Weierstrass transform. The Gaussian function is non-zero for $x \in[-\infty, \infty]$ and would supposedly need an infinite window length. The filter function is supposed to be the kernel of an integral transform. The Gaussian kernel is continuous and is not discrete. The cut-off frequency of the filter can be taken as the ratio between the sample rate $F_{s}$ and the standard deviation $\sigma$.

$$
f_{c}=\frac{f_{s}}{\sigma}
$$

The 1D Gaussian filter is given by the equation:

$$
g(x)=\frac{1}{\sqrt{2 \Pi} \sigma} e^{\frac{-x^{2}}{2 \sigma^{2}}}
$$

g

The impulse response of the 1D Gaussian Filter is

$$
g(x)=\frac{1}{\sqrt{2 \Pi} \sigma} e^{\frac{-\sigma^{2} u^{2}}{2}}
$$

Here in the preprocessing step, the input image is passed through a Gaussian filter which results in reduction of the noise in the input image and also results in obtaining an image fit for further processing. Passing the image through the Gaussian filter also enhances the image quality.

B. Conversion of RGB to Lab colour space Image: A Lab color space [21] is a color-opponent space with dimension $\mathrm{L}$ for lightness and ' $a$ ' and ' $b$ ' for the color-opponent dimensions, based on nonlinearly compressed CIE XYZ color space coordinates. Different from the RGB and CMYK color models, Lab color is developed to approximate the human vision. It aims for perceptual uniformity, and its L component relatively corresponds to human perception of lightness. It is therefore used to make accurate color balance corrections by changing the output curves in the ' $a$ ' and ' $b$ ' components, or to regulate the lightness contrast using the $\mathrm{L}$ component. In RGB or CMYK spaces, which model the output of physical devices instead of the human visual perception, these changes are done with the aid of the corresponding blend modes in the editing application.

\subsection{Initial Segmentation Using proposed clustering algorithm}

After applying the preprocessing steps to the input multispectral satellite image, we get an image fit to be segmented. This image is made of thousands of pixels and to classify this image based on each of this individual pixel is a hectic task and is time consuming. Processing this huge amount of data also results in increase of error rate and the degraded performance of the classifier system. Hence, we cluster the pre-processed image into clusters and then select the centroid of each of these clusters formed for the classification process. This is due to the fact, that each member in a cluster will have almost similar pixel values and differ from the centroid value of the cluster by only a small amount. Hence, this centroid value will represent all the pixels in the clusters. As a result, the classification of a centroid of a cluster will act virtually as classification of all the pixels in the cluster. This result in reducing the number of the inputs to the classifier system which reduce the classifier complexity and also the time incurred. It also results in making the system more efficient and accurate. Here for the clustering, we are using a fuzzy incorporated hierarchical clustering, which is an extension of basic hierarchical clustering.

We have used hierarchical clustering here; as well multiple results from the dendrogram structure of the hierarchical clustering process. We obtain the different number of classifications for different levels as each level will have a unique number of clusters. Here about 20-30 number of clusters after the clustering process is desirable and yields better results. But the use of the normal hierarchical algorithm doesn't yield that a good result and is error prone. These reasons prompted us to extend the basic hierarchical algorithm. In our extension, we have incorporated Fuzzy C Means algorithm and the clustering process is explained below:

1. Given a pre-processed multispectral satellite image. Let it have M pixels. At first, we have to create a pixel difference matrix $\lambda_{i}$, which has the difference in pixel values $\partial_{i j}$ of each pixel to the other pixels in the image. Initially each of the pixel ( $P_{i}$, where $0<i \leq M$ ) act as a different cluster and hence it forms $\mathrm{M}$ clusters. Let the $\mathrm{M}$ clusters of the image be represented as $C_{i}$, where $0<i \leq M$ and the pixel difference matrix is defined by:

$\lambda=\left[\begin{array}{cccccc}\partial_{11} & \partial_{12} & \partial_{13} & \partial_{14} & . . & \partial_{1 M} \\ \partial_{21} & \partial_{22} & \partial_{23} & \partial_{24} & . . & \partial_{2 M} \\ \partial_{31} & \partial_{32} & \partial_{33} & \partial_{34} & . . & \partial_{3 M} \\ \partial_{41} & \partial_{42} & \partial_{43} & \partial_{44} & . . & \partial_{4 M} \\ . . & . . & . . & . . & . . & . . \\ \partial_{M 1} & \partial_{M 2} & \partial_{M 3} & \partial_{M 4} & . . & \partial_{M M}\end{array}\right]$

Where, $\partial_{i j}$ is the difference in pixel value between the $i$ th and $j$ th cluster.

2. From the matrix, which has the pixel difference between the cluster pixel values, we search and 
find out two clusters $\left(C_{i} \& C_{j}\right.$ ) having the minimum pixel difference value and merge the two to form a new cluster $C_{i j}$. New cluster $C_{i j}$ formed will be the one having $\partial_{\min }$ value in the pixel difference matrix.

3. The new cluster $C_{i j}$ is formed from the two individual clusters by a two-step procedure. In the initial step, the centroid pixel value for the new cluster is approximated as means of the pixel values of the individual clusters. That is the new centroid $O_{i j}$ will be:

$$
O_{i j}=\frac{C_{i}+C_{j}}{2}
$$

4. Subsequently, we find out the original centroid pixel value by incorporating the Fuzzy C Means algorithm. This process is done as taking the mean value and will not be that accurate and will give false output results. In-order to find the original centroid pixel value, we first find the membership value and then calculate the modified centroid value using this membership value and the earlier obtained centroid value.

Membership value is given by

$$
\mu_{i j}=\frac{1}{\sum_{k=1}^{C}\left(\frac{\left\|P_{i}-O_{i j}\right\|}{\left\|P_{i}-o_{k}\right\|}\right)^{\frac{2}{m-1}}}
$$

Where, $O_{i j}$ is the approximated centroid pixel value of the new merged cluster found by step no $3, O_{k}$ is the centroid pixel values of the other clusters excluding the newly formed cluster, $\mathrm{m}$ is any positive real number greater than 1.
Modified centroid pixel value of the newly formed cluster

$$
C_{i j} \text { is given by } O_{j}=\frac{\sum_{i=1}^{N} \mu_{i j}^{m} x_{i}}{\sum_{i=1}^{N} \mu_{i j}^{m}}
$$

After this process, $C_{i j}$ will have the modified centroid pixel value and in the subsequent calculations the pixel value of cluster $C_{i j}$ will be known by this value.

5 . The above steps will result in the formation of a new cluster from the two individual clusters with the greatest similarity. Hence it results in the decrease in the total number of clusters by one, after every iteration.

6. The difference pixel matrix is modified and the $C_{i}$ and $C_{j}$ values have been replaced by the respective $C_{i j}$ value. This will lead to reduction of pixel difference matrix dimension from $M \times M$ to $(M-k) \times(M-k)$ after the $k$ loops.

7. Go back to the step number 2 till we have desired number of clusters. A count of 20 to 30 numbers of cluster result in a better classification.

\subsection{Training Data Selection for SVM}

In this section, we discuss the training data selection given to the SVM for the classification purpose. Our proposed technique aims to classify the image into land use and land cover. This is effectively done making use of the colour features in the satellite image. Each of the elements in earth has a colour by which it is distinguished. Hence in-order to classify the image using the SVM, we make use of the colour of these earthly elements. Certain colours in the multispectral image stand for 'land use' and certain for the 'land cover'. We have identified those colours and these colour details are given to the SVM classifier for classification purpose. 


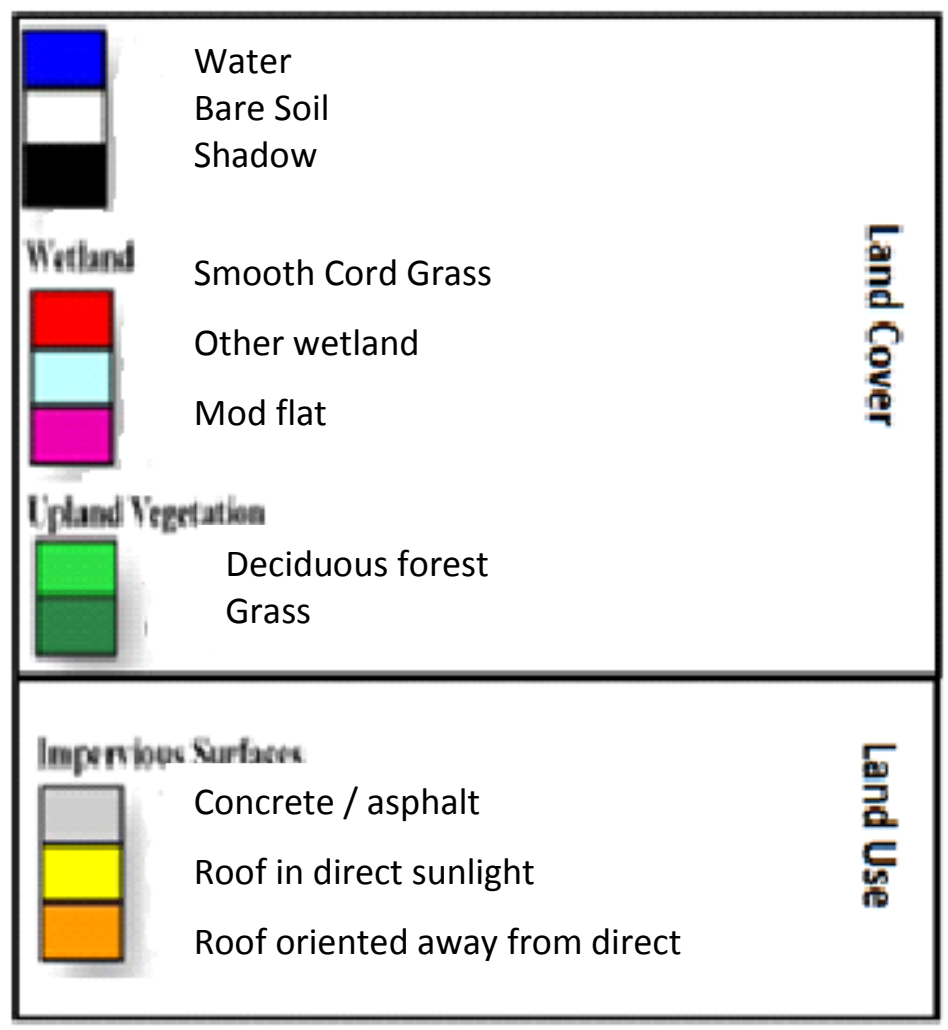

Figure 3. Chart showing colours with which the elements of the earth are represented.

The above figure shows different colours and what they represent in the multispectral image. It also shows what all come under the land use and land cover classification. Some of the elements that come under the land use are concrete buildings, roofs and those in land cover include that of vegetation, soil, mud, crops. These colour details are given to the SVM and based on this data classification is done in the final step.

\subsection{Final Classification Using SVM}

The pre-processed multispectral satellite image is then clustered using the fuzzy incorporated clustering to obtain clusters. Here it can be seen that each member in a cluster will have almost similar pixel values and differ from the centroid value of the cluster by only a small amount. Hence the centroid value can represent all the pixels in the clusters. Hence, by performing single step of classifying the centroid of a cluster will act like as multiple steps of classifying all the pixels in the cluster. This result in reducing the number of the inputs to the classifier system which reduce the classifier complexity and also the time incurred.

Suppose we consider the $i^{\text {th }}$ cluster having $\mathrm{n}$ elements where each pixel having a value of $P_{k}$. Then, the centroid value of the $i^{\text {th }}$ cluster, $O_{i}$ can be calculated as $O_{i}=\frac{\sum_{k=1}^{n} P_{k}}{n}$. Similarly, repeat the process for all the

clusters to obtain the centroid values for each of the clusters. Suppose there are $\mathrm{N}$ numbers of cluster, then centroid set $O=\left\{O_{1}, O_{2}, O_{3}, \ldots \ldots ., O_{N}\right\}$, will be given as the input to the SVM classifier. This centroid set is classified based on the trained data given to the SVM before and we obtain the classified result as land use and land cover.

\section{RESULTS AND DISCUSSION}

The proposed technique for classification of satellite images is implemented in MATLAB. In this section, we discuss and analyse the proposed approach. The sub-section 4.1 describes the overall experimentation and the results of the proposed approach. And, in the section 4.2, the evaluation results are presented which shows that the proposed approach is more efficient compared to the baseline techniques.

\subsection{Experimental results}

In this section, we discuss the results of the proposed technique. We have used multispectral satellite image as the input image which is to be classified as land use and land cover. In our proposed classification technique, initially preprocessing is done where the input image is subjected to a set of pre-processing steps is carried out so that the image gets transformed suitably for segmentation. It consists of Gaussian filtering and conversion of RGB to Lab colour space image. The pre-processed image is segmented using the fuzzy incorporated hierarchical clustering algorithm. Training data selection is carried out for SVM and finally, classification of the multispectral satellite images using SVM is done based on the trained data and the centroid pixel values. Figure 4 shows the input satellite image taken for experimentation and the figure 4 shows the trained SVM graph. Along with, figure 6 shows the final classified output for the input satellite image. 


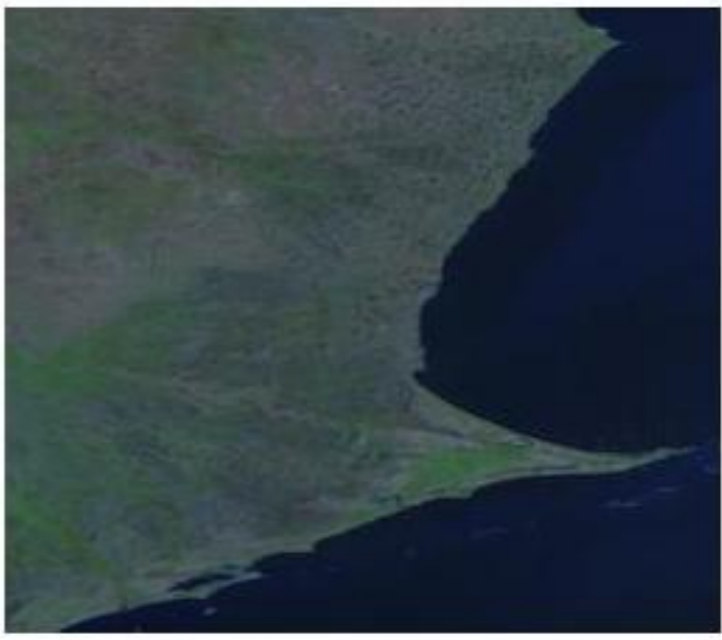

Figure 4. Input satellite image

The above figure (4) shows the input multispectral image of an area taken from the satellite and we can we clearly see the land and water features. Our aim is to classify the image into land use and land cover using the proposed technique.

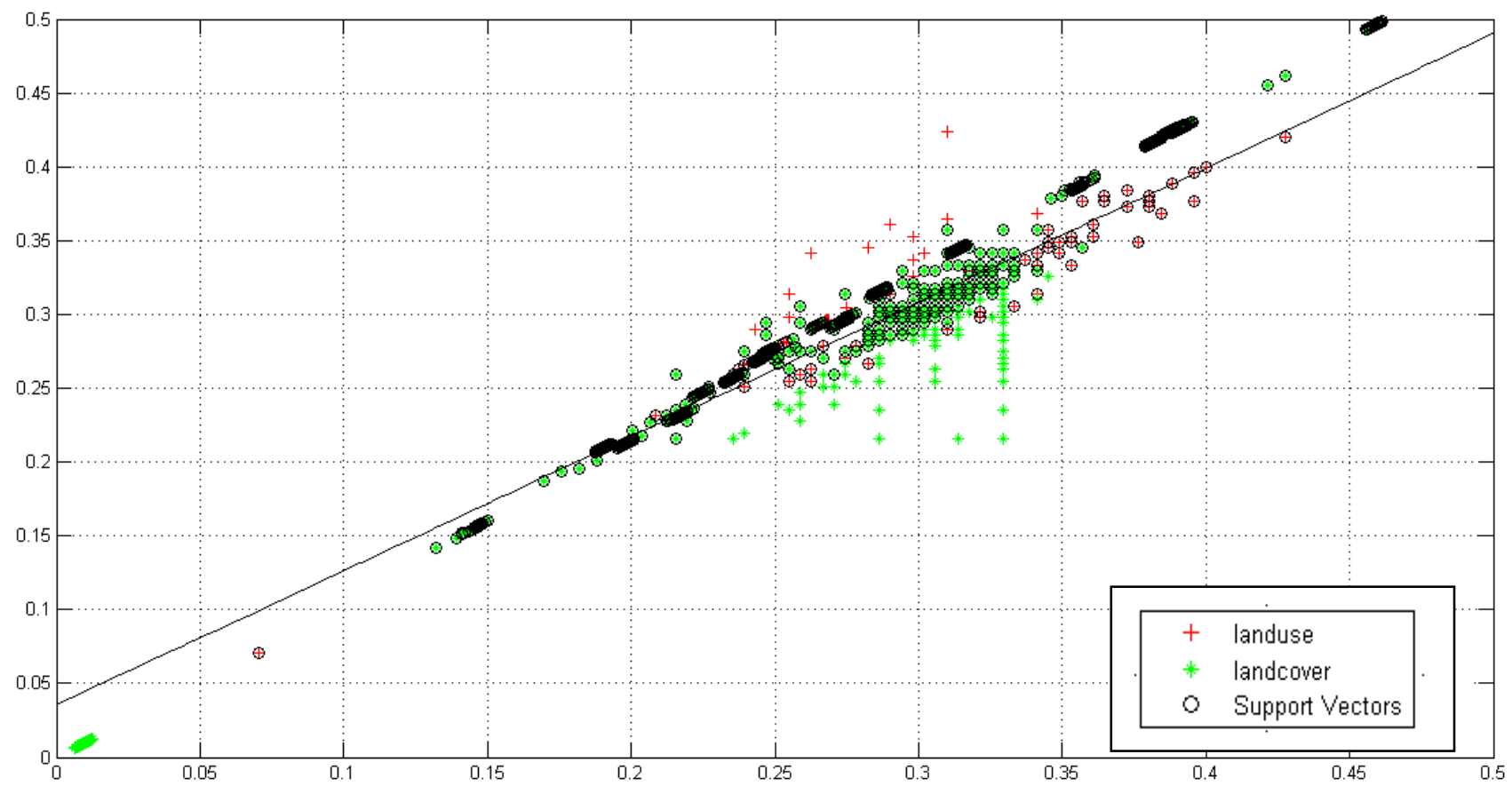

Figure 5. The plot of trained SVM

The above figure (5) shows the plot of the trained SVM.

Here we can see the hyperplane which classifies the input image into land use and land cover. We can also see the points near to the hyperplane indicated by circle spots. We can also see the classified output points into land use and land cover where land use is represented by green and land use by red. 


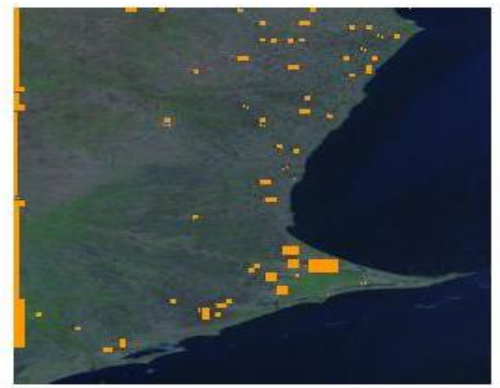

Figure 6. Classified image

The above figure 6 shows the final classified image from the input image. Here we can see that the land use areas are represented by the yellow blocks and land cover areas are unchanged.

\subsection{Performance evaluation}

In this section, we present the performance evaluation results of the proposed technique. Here we have evaluated and compared the results with various clustering algorithms and also with various classifiers. The first part in this section, deals with the comparison with various clustering algorithms. In the later part, evaluation and comparison is made by comparing various classifiers. A detailed analysis, followed by the evaluation graph is made in each part. Analysis made and results obtained clearly demonstrate the efficiency of the proposed approach in classifying the multispectral image into land use and land cover regions.

\subsubsection{Evaluation using different clustering algorithm \\ For performance evaluation, the proposed technique (proposed clustering $+\mathrm{SVM}$ ) is evaluated with the traditional clustering algorithm like, FCM clustering + SVM and Hierarchical clustering + SVM. Furthermore, rather than the SVM classifier, neural network network-based classifier is also used to extensively analyse the results. The accuracy value is computed by dividing the total number of similar pixels identified as land use to the number of pixels in the land use region. The following graphs and tables}

signify the performance of the technique compared with the traditional methods.

Table 1. Accuracy of the different methods in land use classification

\begin{tabular}{|c|c|c|c|}
\hline Techniques & \multicolumn{3}{|c|}{ Number of similar pixels } \\
\hline \begin{tabular}{c} 
No. of clusters \\
\hline $\begin{array}{c}\text { Proposed } \\
\text { clustering + } \\
\text { SVM }\end{array}$
\end{tabular} & 1560 & 84 & 8 \\
\hline $\begin{array}{c}\text { FCM+SVM } \\
\text { Hierarchical + } \\
\text { SVM }\end{array}$ & 5 & 20 & 106 \\
\hline
\end{tabular}

Table 2. Accuracy of the different methods in land cover classification

\begin{tabular}{|c|c|c|c|}
\hline Techniques & \multicolumn{3}{|c|}{ Number of similar pixels } \\
\hline No. of clusters & Cluster 1 & Cluster 2 & Cluster 3 \\
\hline $\begin{array}{c}\text { Proposed } \\
\text { clustering + } \\
\text { SVM }\end{array}$ & 163580 & 165056 & 165132 \\
\hline FCM+SVM & 165135 & 165120 & 165034 \\
\hline $\begin{array}{c}\text { Hierarchical + } \\
\text { SVM }\end{array}$ & 131390 & 141490 & 135390 \\
\hline
\end{tabular}

Here, table 1 and table 2 shows the accuracy of the different methods in land use and land cover classification. Here we compare our proposed clustering technique with that of FCM and hierarchical techniques. Here the results obtained are using the respective clustering technique with the use of the SVM classifier. The number of similar pixels is calculated and given in the above tables. We can observe from the above tables that our proposed technique achieves the best results both in land use and land cover classification. 


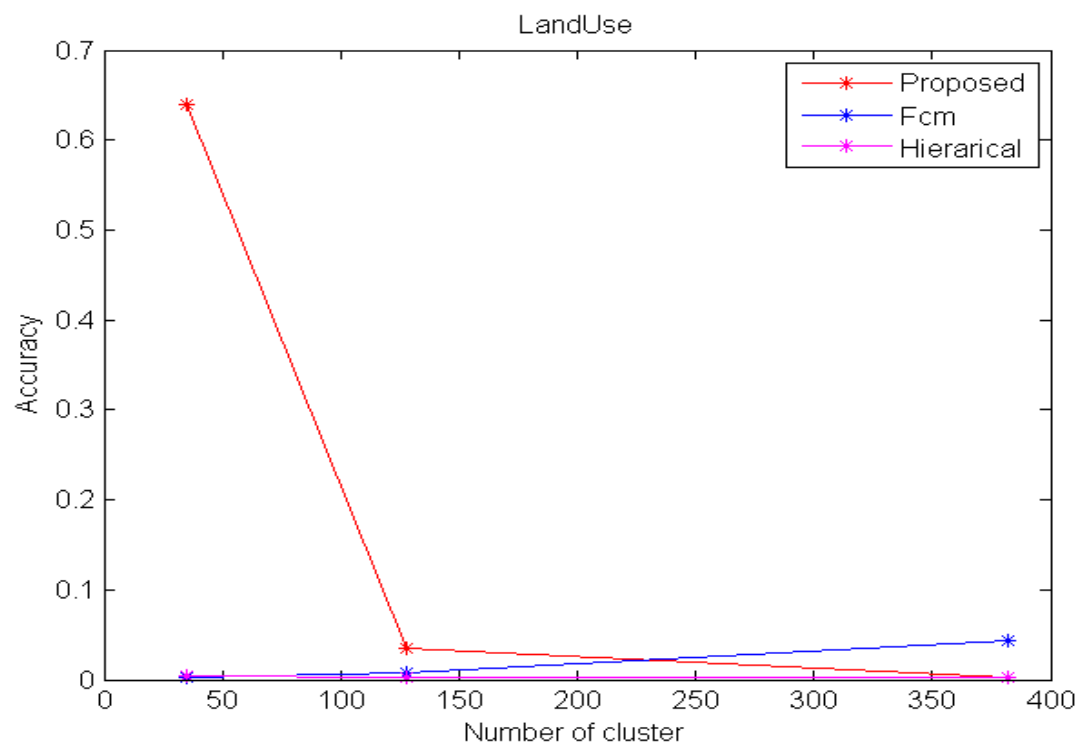

Figure 7. Accuracy graph-land use classification using SVM

Figure 7 shows the plot of the accuracy graph considering the land use classification using SVM classifier. Here, we can see that initially, when the number of clusters is low, our proposed technique achieve very good accuracy and yields a better result when compared to the performance of the other techniques. We can also see a decrease in the accuracy as the cluster number increases.

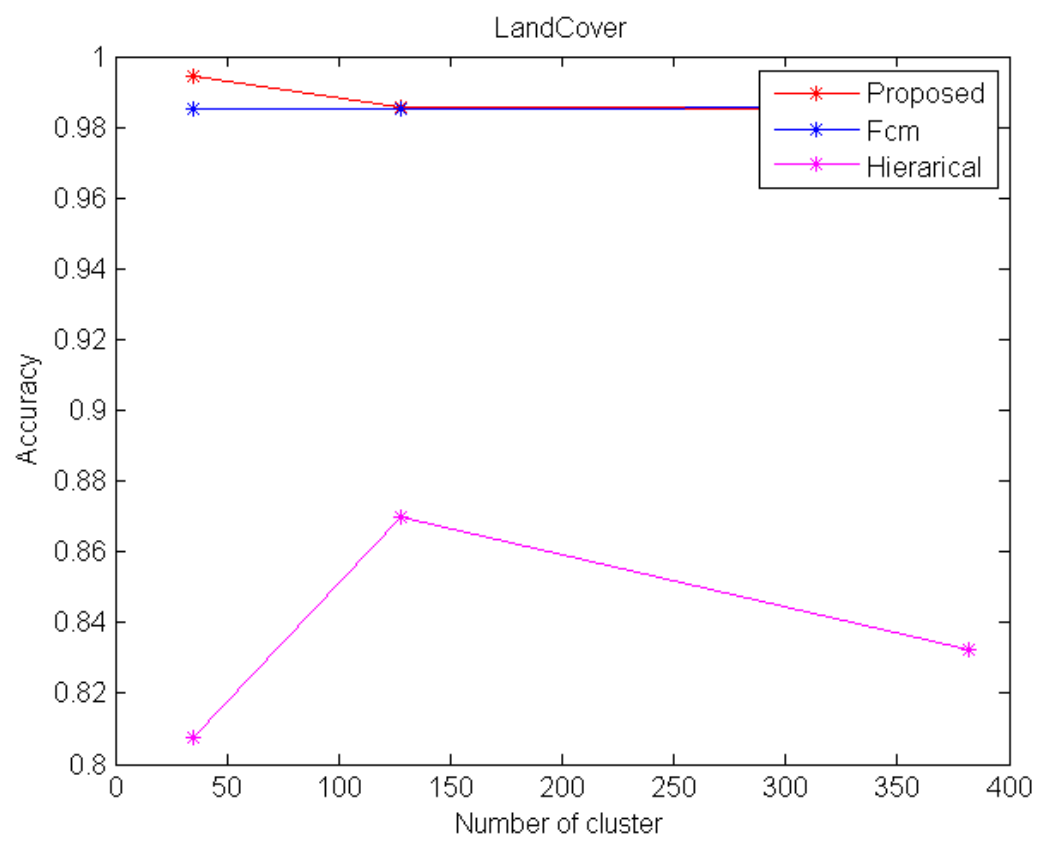

Figure 8. Accuracy graph-land cover classification using SVM

The accuracy plot of the proposed technique for the land cover classification is given in figure 8 . We infer that both our technique and the FCM achieve very high values when compared to the hierarchical clustering. As the cluster size increases, the accuracy value goes down to a certain level and then becomes constant irrespective of the cluster size. Here we can see that our proposed technique achieves a best peak accuracy value of $99.5 \%$ when compared to the FCM which has peak value of $98.8 \%$ and Hierarchical which has a peak value of $87 \%$. 


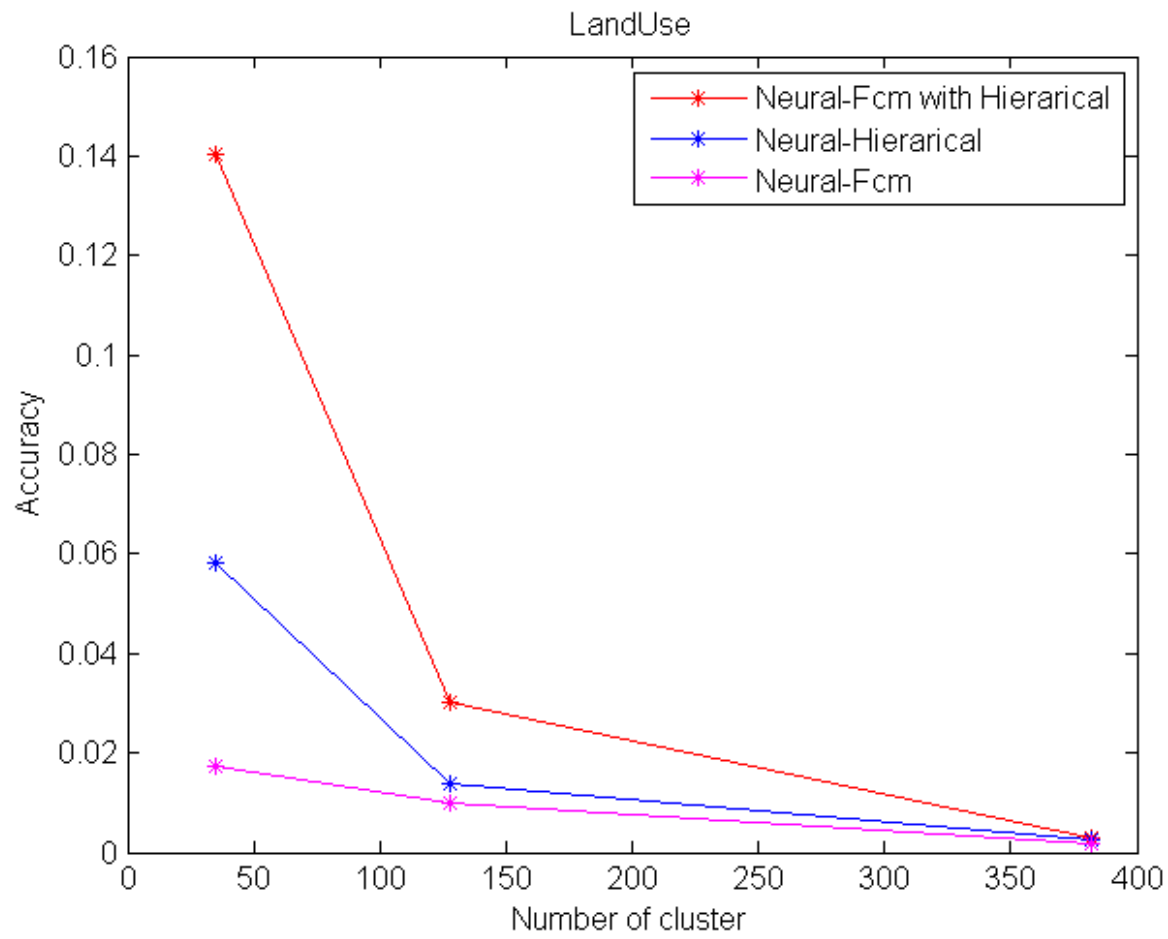

Figure 9. Accuracy graph-land use classification using neural network

Subsequently, we consider the techniques incorporating the neural networks classifier instead of the SVM. And here, the comparison is made of the clustering techniques making use of the neural networks. Figure 9 shows the plot of accuracy for the land use classification using neural network. Use of neural network instead of the SVM has resulted in a clear decrease in the accuracy value of the proposed technique.
From the figure, we can see that the proposed clustering technique achieves best results when compared to other clustering techniques. As the number of clusters increases the accuracy value is shown to be decreasing. The employment of neural network instead of the SVM has resulted in decreasing the peak accuracy value form $66 \%$ to $14 \%$ in the land use classification.

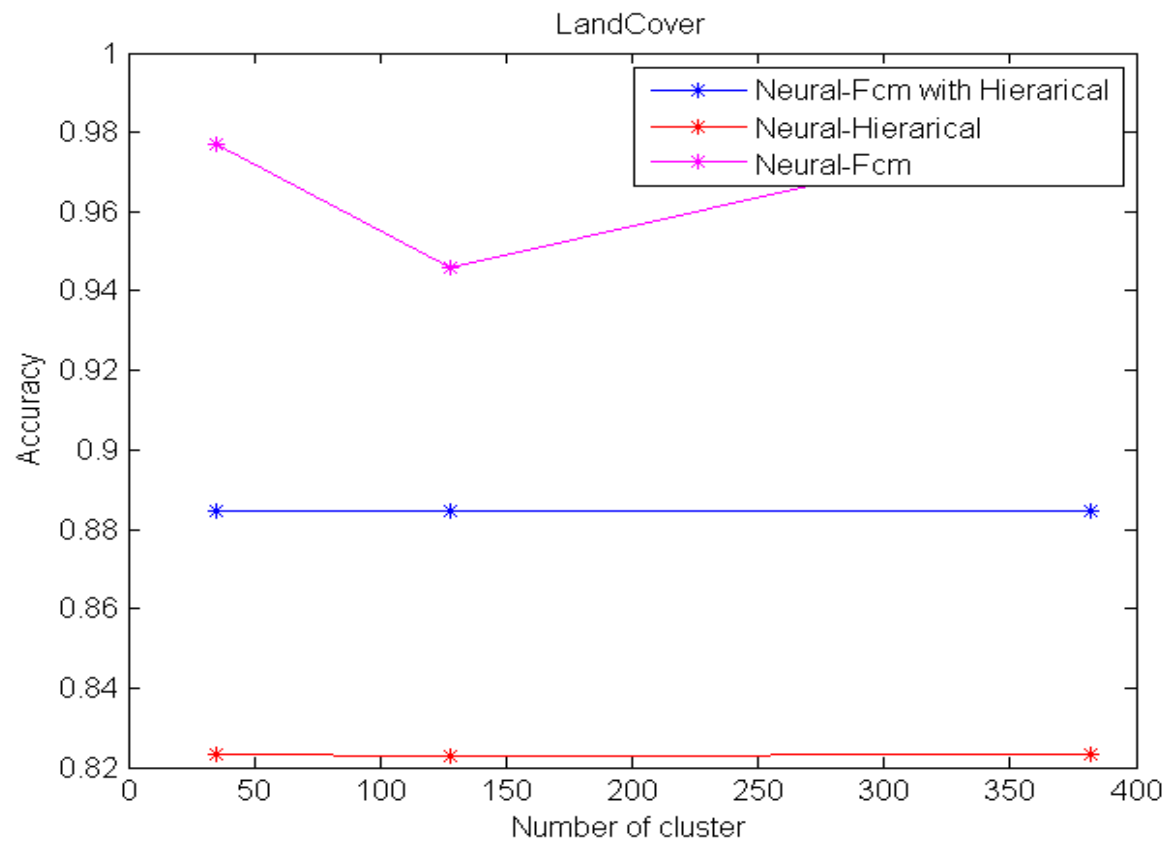

Figure 10. Accuracy graph-land cover classification using neural network 
Figure 10 shows the plot of accuracy of land cover classification using neural networks. Here we can see that the proposed clustering techniques a good accuracy of $88.2 \%$ irrespective of the cluster size. Here also the use of neural network instead of the SVM has resulted in decreasing the peak accuracy value form $99.5 \%$ to $88.2 \%$ in the land use classification.

\subsubsection{Evaluation of classifier's performance}

For performance evaluation, the proposed technique (proposed clustering + SVM) is evaluated with the networkbased approach. The accuracy value is computed by dividing the total number of similar pixels identified as land use to the number of pixels in the land use region. The following graphs signify the performance of the proposed technique compared with the neural network. Figure 11 shows the plot of the accuracy of land cover classification using the two classifiers SVM and the neural network. Figure 12 shows the plot of the accuracy of land cover classification using the two classifiers SVM and the neural network. In both the cases it can be seen that our proposed technique which uses SVM, achieves best results when compared to use of the neural networks. Use of neural instead of the SVM has resulted in decreasing the accuracy value in both the land use and land cover classification. In land cover classification, the peak accuracy value has decreased from $99.5 \%$ to $88.2 \%$ whereas in land use classification it has resulted in decreasing the peak accuracy value form $66 \%$ to $14 \%$. These results clearly indicate that use of SVM results in attainment of better accuracy level.

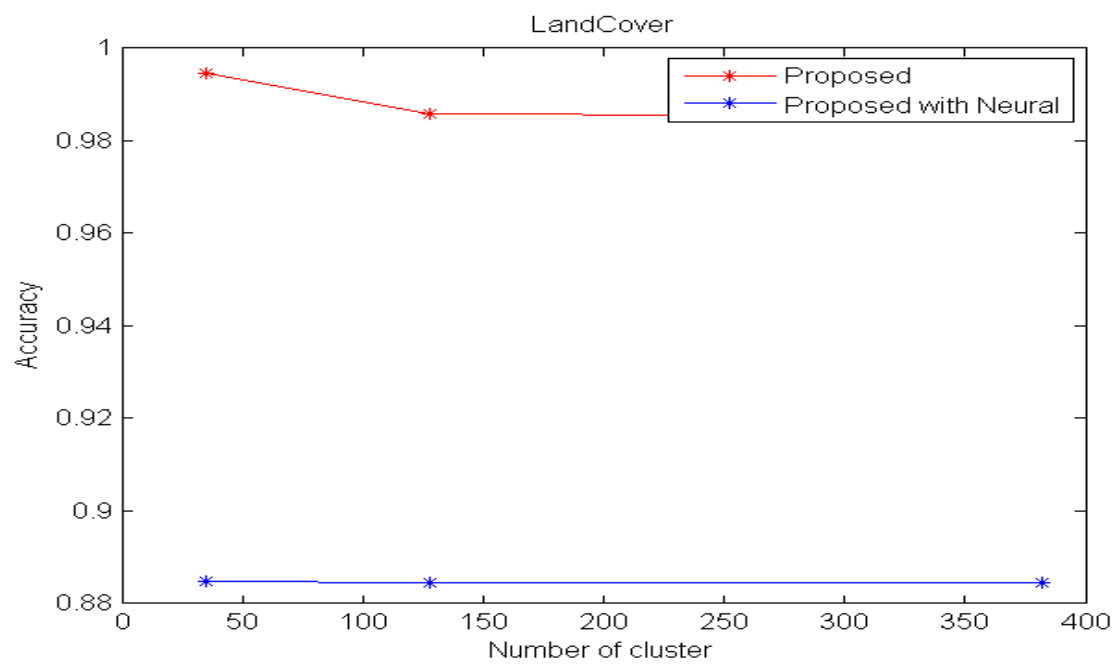

Figure 11. Accuracy graph-land cover classification

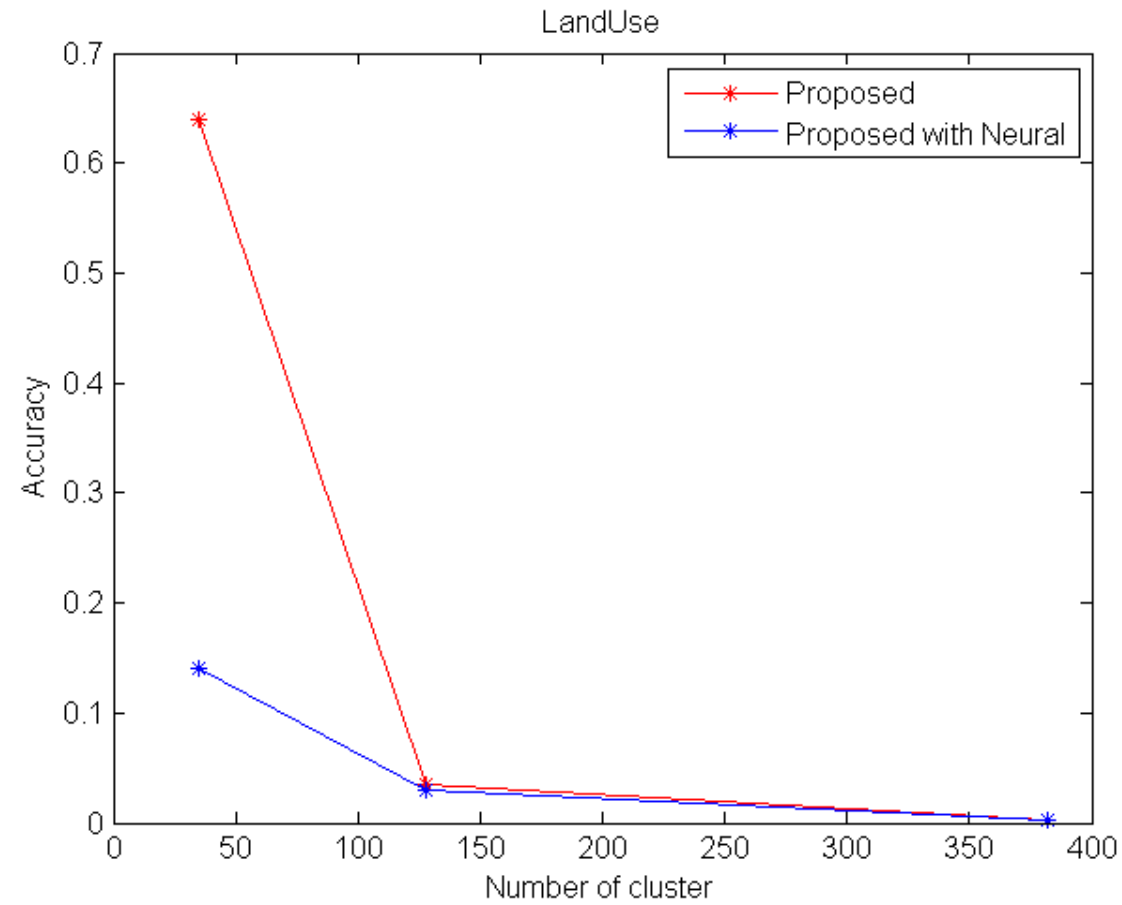

Figure 12. Accuracy graph-land use classification 


\section{CONCLUSION}

In this paper, we have proposed an efficient image classification technique for multispectral remote sensed satellite images with the aid of clustering and Support Vector Machines (SVM). Here in our proposed classification technique is made of four phases namely preprocessing, segmentation, training of SVM and final classification using SVM. In the pre-processing step, the input image is subjected a set of pre-processing steps which includes Gaussian filtering and conversion of RGB to Lab color space image. The pre-processing results in transforming the input image into an image fit for segmentation. After the preprocessing, the image is segmented for which we have used the fuzzy incorporated hierarchical clustering algorithm. This result in the image being segmented into clusters. SVM is trained according to the data given. Finally the image is given as input to the trained SVM, which classifies the multispectral satellite images into land use and land cover regions according to the trained data and pixel values. As a result we get a classified image. The experimental results have demonstrated the effectiveness of the proposed classification technique in classifying into land cover and land use regions. The experimentation is carried out using the multi-spectral satellite images and the analysis ensures that the performance of the proposed technique is improved compared with traditional clustering algorithm. In future, we aim to extent our approach into classifying the multispectral image into multiple regions rather than just land use and land cover. In that case, we will be able to distinguish between land features in a better way and can be more useful.

\section{REFERENCES}

[1] K Perumal and R Bhaskaran, "SVM-Based Effective Land Use Classification System For Multispectral Remote Sensing Images", (IJCSIS) International Journal of Computer Science and Information Security, Vol. 6, No. 2, pp.95-107, 2009.

[2] Jan Knorn, Andreas Rabe, Volker C. Radeloff, Tobias Kuemmerle, Jacek Kozak, Patrick Hostert, "Land cover mapping of large areas using chain classification of neighboring Landsat satellite images", Remote Sensing of Environment, Vol. 118, pages 957-964 , 2009.

[3] Xiaochen Zou, Daoliang Li, "Application of Image Texture Analysis to Improve Land Cover Classification", WSEAS Transactions on Computers, Vol. 8, No. 3, pp. 449-458, March 2009.

[4] Reda A. El-Khoribi, "Support Vector Machine Training of HMT Models for Multispectral Image Classification", IJCSNS International Journal of Computer Science and Network Security, Vol.8, No.9, pp.224-228, September 2008.

[5] B Sowmya and B Sheelarani , "Land cover classification using reformed fuzzy C-means", Sadhana, Vol. 36, No. 2, pp. 153-165, 2011.

[6] V.K.Panchal, Parminder Singh, Navdeep Kaur and Harish Kundra, "Biogeography based Satellite Image Classification", International Journal of Computer Science and Information Security IJCSIS, Vol. 6, No. 2, pp. 269-274, November 2009.

[7] Huang B, Xie C, Tay R, Wu B, 2009, "Land-use-change modeling using unbalanced support-vector machines", Environment and Planning B: Planning and Design , Vol.36, No.3, pp.398-416,2009.
[8] James A. Shine and Daniel B. Carr, "A Comparison of Classification Methods for Large Imagery Data Sets", JSM 2002 Statistics in an ERA of Technological Change-Statistical computing section, New York City, pp.3205-3207, 11-15 August 2002.

[9] D. Lu, Q. Weng, "A survey of image classification methods and techniques for improving classification performance", International Journal of Remote Sensing, Vol. 28, No. 5, pp. 823-870, January 2007.

[10] M. Govender, K. Chetty, V. Naiken and H. Bulcock, "A comparison of satellite hyperspectral and multispectral remote sensing imagery for improved classification and mapping of vegetation", Water SA, Vol. 34, No. 2, April 2008.

[11] Jasinski, M. F., "Estimation of subpixel vegetation density of natural regions using satellite multispectral imagery", IEEE Transactions on Geoscience and Remote Sensing, Vol. 34, pp. 804-813, 1996.

[12] C. Palaniswami, A. K. Upadhyay and H. P. Maheswarappa, "Spectral mixture analysis for subpixel classification of coconut", Current Science, Vol. 91, No. 12, pp. 1706 -1711, 25 December 2006.

[13] Ming-Hseng Tseng, Sheng-Jhe Chen, Gwo- Haur Hwang, Ming-Yu Shen, "A genetic algorithm rulebased approach for land-cover classification", Journal of Photogrammetry and Remote Sensing ,Vol.63, No.2, (3), pp. 202-212, 2008.

[14] Pall Oskar Gislason, Jon Atli Benediktsson, Johannes R. Sveinsson, "Random Forests for land cover classification", Pattern Recognition Letters,Vol.27, No.4, (3), pp. 294-300, 2006.

[15] Hua-Mei Chen, Varshney, P.K. and Arora, M.K, "Performance of mutual information similarity measure for registration of multitemporal remote sensing images “, IEEE Transactions on Geoscience and Remote Sensing, Vol.41 No.11, pp. 2445 - 2454, 2003.

[16] Cristianini, Nello and Shawe-Taylor, John, "An Introduction to Support Vector Machines and other kernel based learning methods", Cambridge University Press, Cambridge, 2000.

[17] Li Zhuo, Jing Zheng, Fang Wang, Xia Li, Bin Ai, Junping Qian, "A Genetic Algorithm Based Wrapper Feature Selection Method For Classification Of Hyperspectral Images Using Support Vector Machine", The International Archives of the Photogrammetry, Remote Sensing and Spatial Information Science, Vol. XXXVII, No. B7, pp.397-402, 2008.

[18] S. C. Johnson, "Hierarchical Clustering Schemes", Psychometrika, Vol.2, pp.241-254, 1967.

[19] J. C. Dunn (1973): "A Fuzzy Relative of the ISODATA Process and Its Use in Detecting Compact WellSeparated Clusters", Journal of Cybernetics, Vol. 3, pp.32-57, 1973.

[20] R.A. Haddad and A.N. Akansu, "A Class of Fast Gaussian Binomial Filters for Speech and Image Processing," IEEE Transactions on Acoustics, Speech and Signal Processing, vol. 39, pp 723-727, March 1991.

[21] Hunter and Richard Sewall ,"Accuracy, Precision, and Stability of New Photo-electric Color-Difference Meter", Proceedings of the Thirty-Third Annual Meeting of the Optical Society of America, Vol. 38(12), 1948. 